МОЩНЫЕ ШИРОКОПОЛОСНЫЕ ТВЕРДОТЕЛЬНЫЕ ИМПУЛЬСНЫЕ СВЧ-УСИЛИТЕЛИ МОЩНОСТИ С ВЫСОКОЙ АМПЛИТУДНО-ФАЗОВОЙ
СТАБИЛЬНОСТЬЮ

\author{
Б. В. Емельянов
}

AO «НПП «Пульсар», 105187, Москва, Окружной проезд, 27

\begin{abstract}
Показаны принципы измерения амплитудно-фазовой стабильности. Рассматриваются фактоpbl, ухудшающие амплитудно-фазовую стабильность твердотельных усилителей мощности. Показаны результаты теоретических расчётов и математического моделирования зависимости стабильности от различных факторов. По результатам математического моделирования выявлены основные факторы, ухудшающие амплитудно-фазовую стабильность. Представлены результаты практических измерений стабильности. Предложены варианты, улучшающие амплитудно-фазовую стабильность мощных твердотельных усилителей мощности.
\end{abstract}

Ключевые слова: амплитудно-фазовая нестабильность, импульсный усилитель мощности, саморазогрев транзистора, селекиия движущиися иелей, амплитудная стабильность, фазовая стабильность

Сведения об авторах: Емельянов Борис Вячеславович, emelyanov_bv@pulsarnpp.ru

\title{
HIGH POWER BROADBAND PULSED MICROWAVE SOLID-STATE POWER AMPLIFIER WITH HIGH AMPLITUDE-PHASE STABILITY
}

\author{
B. V. Emelyanov \\ S\&PE Pulsar JSC, 105187 Moscow, Okruzhnoy pr., 27
}

In this paper the principles of measuring the amplitude-phase stability are described. Fa ctors that negatively affect the amplitude-phase stability of a solid-state power amplifier are considered. The results of theoretical calculations and mathematical modeling of the dependence of this stability on various factors are presented, and on the basis of these results the main factors that negatively affect the amplitude-phase stability are identified. In the conclusion I present the results of practical measurements of the stability, and suggest methods to improve the amplitude-phase stability of high-power solid-state power amplifiers.

Keywords: amplitude-phase instability, pulsed power amplifier, transistor self-heating, moving target indication, amplitude stability, phase stability

Data on author: Emelyanov Boris Vyacheslavovich,emelyanov_bv@pulsarnpp.ru 
Для корректной работы системы селекции движущихся целей (СДЦ) [1] радиолокационных станций (РЛС) необходимо, чтобы амплитуда и фаза последовательных радиоимпульсов оставались неизменными от импульса к импульсу. Флуктуация амплитуды и фазы сигнала с выхода передатчика ухудшает подавление сигналов от неподвижных целей в РЛС.

Для установления критерия влияния флуктуации сигнала выходных усилительных модулей РЛС на систему СДЦ и критерия годности передатчика введён параметр, определяющий стабильность выходного сигнала с усилителя, - амплитудно-фазовая нестабильность.

Амплитудно-фазовая нестабильность параметр, отображающий уровень изменения амплитуды и фазы выходных радиоимпульсов от импульса к импульсу. Чем выше данный параметр, тем большее влияние на подавление неподвижных целей привносит модуль. Для большинства усилителей, изготовляемых для РЛС в АО «НПП «Пульсар», амплитудно-фазовая нестабильность должна составлять не более -50 дБ.

Для определения амплитудно-фазовой нестабильности необходимо произвести накопление амплитуд и фаз последовательных радиоимпульсов в определённой точке от начала каждого импульса [2]. Данную информацию можно получить при помощи векторного приёмника. Далее необходимо обработать массив данных для получения численного результата. Существуют три основных алгоритма обработки массива.

\section{Вариант 1. Метод оценки нестабиль- ности по отклонению амплитуды и фазы от среднего значения}

Данный метод оценивает стабильность сигнала по отклонению амплитуды и фазы импульса от среднего значения за определённый период накопления [3].
Фазовая стабильность описывается формулой

$$
S_{\varphi}=-10 \log \frac{1}{N} \sum_{i=1}^{N}\left(\varphi_{i}-\varphi_{c p}\right)^{2},
$$

где $\varphi_{c p}=\frac{1}{N} \sum_{i=1}^{N} \varphi_{i}, \quad \varphi_{i}-$ фаза импульса $N$ - количество накопленных импульсов.

Амплитудная стабильность описывается формулой

$$
S_{A}=-10 \log \left[\frac{\frac{1}{N} \sum_{i=1}^{N}\left(A_{i}-A_{c p}\right)^{2}}{A_{c p}{ }^{2}}\right],
$$

где $A_{c p}=\frac{1}{N} \sum_{i=1}^{N} A_{i}, A_{i}-$ амплитуда импульса, $N$ - количество накопленных импульсов.

Амплитудно-фазовая стабильность рассчитывается по следующей формуле:

$$
S=-10 \log \left(10^{-\frac{S_{A}}{10}}+10^{-\frac{S_{\varphi}}{10}}\right) .
$$

\section{Вариант 2. Метод оценки нестабиль- ности по отклонению амплитуды и фазы от импульса к импульсу [4]}

Фазовая стабильность по данному алгоритму оценивается по следующей формуле:

$$
S_{\varphi}=-10 \log \frac{1}{N-1} \sum_{j=1}^{N-1}\left(\varphi_{j+1}-\varphi_{j}\right)^{2} .
$$

Амплитудная стабильность по данному алгоритму оценивается по следующей формуле:

$$
S_{A}=-10 \log \left[\frac{\frac{1}{N-1} \sum_{j=1}^{N-1}\left(A_{j+1}-A_{j}\right)^{2}}{A_{c p}{ }^{2}}\right]
$$

Общая стабильность в данном методе также описывается формулой (3). 


\section{Вариант 3. Метод спектрального анализа [2]}

При реализации рассматриваемого метода после накопления массива амплитуд и фаз проводится дискретное преобразование Фурье данного массива и измеряется отношение уровня основной гармоники к сумме уровней побочных гармоник. Данное отношение является уровнем амплитудно-фазовой нестабильности.

Первый метод наименее точный, но наиболее быстрый в вычислениях. Поскольку система СДЦ измеряет изменение между последовательными импульсами, а не отклонение каждого импульса от среднего, актуальнее использовать метод 2 или метод 3. В дальнейших расчётах будет применяться третий метод, поскольку он точнее остальных методов коррелирует с коэффициентом улучшения СДЦ в РЛС заказчика.

\section{Теоретический расчёт влияния основных факторов на амплитудно- фазовую нестабильность}

Факторы, влияющие на нестабильность радиоимпульсов, можно разделить на два типа.

1. Детерминированные факторы. Влияние данных факторов на амплитуду и фазу радиоимпульсов можно описать функцией, зависящей от расстановки выходных радиоимпульсов [2]. К детерминированным факторам можно отнести саморазогрев транзистора, просадку и восстановление напряжения питания транзисторов и эффект ловушек у транзисторов с высокой подвижностью носителей заряда (транзисторы на арсениде и нитриде галлия).

2. Недетерминированные факторы. Влияние данных факторов не зависит от расстановки выходных радиоимпульсов. К недетерминированным факторам можно отнести собственные шумы активных и пассивных элементов цепи, шум источника питания транзисторов, шум и флуктуации сигналов управляющих элементов усилителя и самовозбуждение транзисторов.

По результатам экспериментальных исследований выявлено, что на сигналы с переменным периодом повторения основное влияние оказывают детерминированные факторы, а в случае сигналов, использующих моноимпульсную последовательность, детерминированные факторы не ухудшают стабильность выходных радиоимпульсов.

Поскольку большинство усилителей, производимых в АО «НПП «Пульсар», работает с сигналами с переменным периодом повторения радиоимпульсов, будет рассчитано влияние основных детерминированных факторов, воздействующих на амплитудно-фазовую нестабильность. Для расчёта использована модель биполярного транзистора [5] со следующими параметрами: выходная импульсная мощность 200 Вт, рабочая частота - от 2,7 до 2,9 ГГц, напряжение питания - $41 \mathrm{~B}$, коэффициент усиления -8 дБ, входная импульсная мощность -35 Вт.

\section{Саморазогрев транзистора}

Температура перехода транзистора зависит от теплоёмкости и теплопроводности различных слоёв и элементов транзистора. Поскольку данные структуры имеют различную теплоёмкость и теплопроводность, рост температуры во время радиоимпульса нелинеен. Тепловые параметры транзистора можно описать при помощи эквивалентной схемы из нескольких последовательно соединённых $r c$-цепочек. Каждая $r c$-цепочка соответствует определённой структуре транзистора, сопротивление $r c$-цепочки характеризует теплопроводность, а номинал конденсатора - теплоёмкость.

За время паузы между импульсами температура перехода может не успеть вернуться к исходной. В случае переменного периода повторения разная температура перехода в момент начала радиоимпульса 


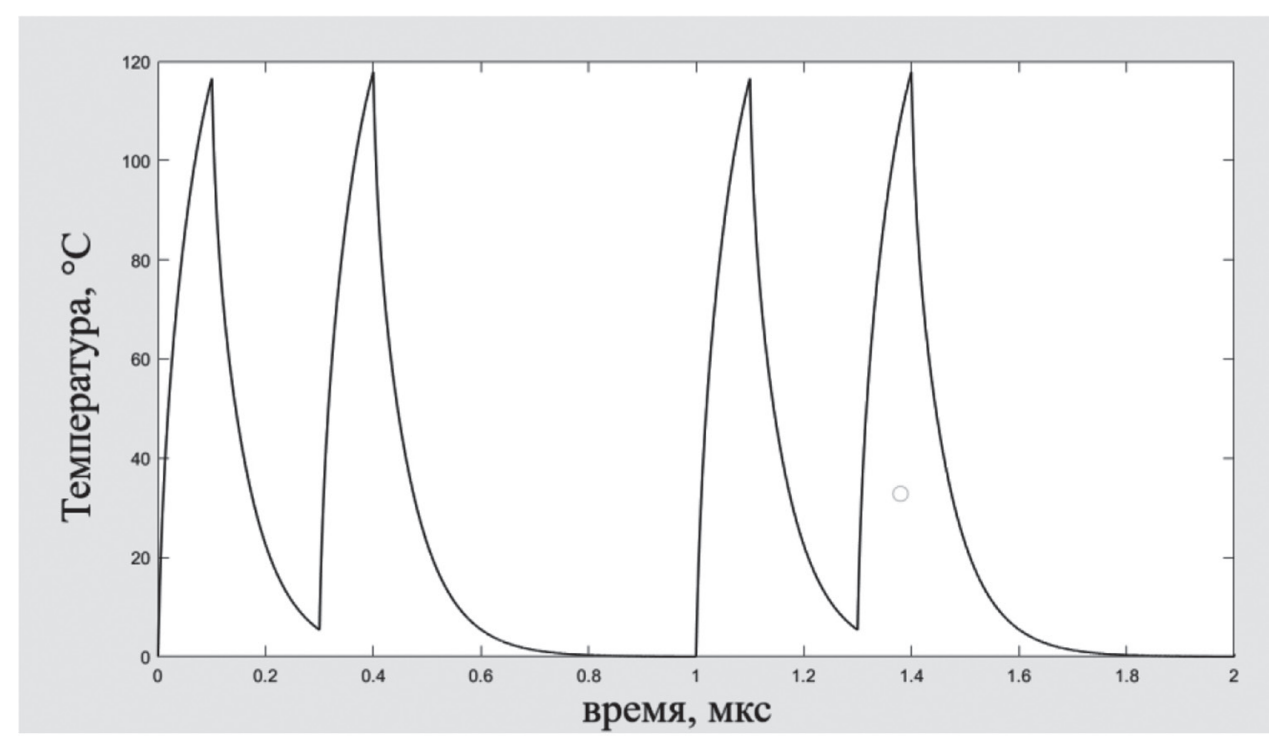

Puc. 1

Зависимость температуры перехода транзистора от времени

будет влиять на стабильность выходного сигнала, поскольку температура транзистора влияет на амплитуду и фазу выходного сигнала каскада. Для анализа зависимости стабильности от теплового сопротивления транзистора написана программа, позволяющая теоретически рассчитать температуру транзистора во времени. На рис. 1 показаны результаты моделирования саморазогрева транзистора при импульсном сигнале.

Далее расчётная температура подставляется в программу моделирования транзисторов для расчёта амплитуды и фазы сигнала. Проведён расчёт амплитудно-фа- зовой нестабильности при различных значениях теплового сопротивления транзисторов. Результаты представлены в табл. 1.

На основании полученных результатов можно сделать вывод, что при линейном росте теплового сопротивления также линейно возрастает амплитудно-фазовая нестабильность. При этом уровень амплитудной нестабильности практически идентичен уровню амплитудно-фазовой нестабильности. Данная закономерность свидетельствует о том, что повышение теплового сопротивления не оказывает существенного влияния на уровень фазовой стабильности.

Таблица 1

Зависимость нестабильности от изменения импульсного теплового сопротивления транзистора

\begin{tabular}{|c|c|c|}
\hline $\begin{array}{c}\text { Тепловое сопротивление, } \\
\text { К/Вт }\end{array}$ & $\begin{array}{c}\text { Амплитудная } \\
\text { нестабильность, дБ }\end{array}$ & $\begin{array}{c}\text { Амплитудно-фазовая } \\
\text { нестабильность, дБ }\end{array}$ \\
\hline 0,2 & $-67,8$ & $-67,7$ \\
\hline 0,4 & $-62,8$ & $-62,8$ \\
\hline 0,6 & $-58,9$ & $-58,8$ \\
\hline 0,8 & $-55,7$ & $-55,6$ \\
\hline 1,0 & $-53,3$ & $-53,3$ \\
\hline
\end{tabular}


Нестабильность напря⿻ения питания усилительных каскадов

Флуктуация напряжения питания каскада обусловлена конструкцией источника питания, характером нагрузки на блок питания и накопительной ёмкостью.

Уровень напряжения питания транзистора влияет на выходную мощность и фазу выходного сигнала транзистора. Для определения зависимости стабильности сигнала от уровня флуктуации напряжения питания проведён расчёт амплитуды и фазы сигнала при различных уровнях напряжения. Далее составлен массив из комплексных чисел с последовательным чередованием значений амплитуды и фазы выходного сигнала усилителя при номи- нальном уровне напряжения сигнала и при напряжении, сдвинутом на прогнозируемый уровень флуктуации напряжения.

Проведён расчёт амплитудно-фазовой стабильности каскада с девиацией напряжения при различном согласовании выходной цепи транзистора. В табл. 2 указаны зависимости амплитудно-фазовой нестабильности от изменения уровня флуктуации напряжения питания при различных режимах работы транзистора. Можно сделать вывод, что в линейном режиме нестабильность ниже, чем в режиме насыщения. Но линейный режим не является оптимальным с точки зрения коэффициента полезного действия и выходной мощности для импульсных усилителей мощности РЛС.

Таблица 2

Зависимость нестабильности от флуктуации напряжения питания в режиме насыщения и линейном режиме работы транзистора

\begin{tabular}{|c|c|c|c|c|}
\hline \multirow[b]{2}{*}{$d U, \mathrm{~B}$} & \multicolumn{2}{|c|}{ Режим насыщения } & \multicolumn{2}{|c|}{ Линейный режим } \\
\hline & $\begin{array}{c}\text { Амплитудная } \\
\text { нестабильность, } \\
\text { дБ }\end{array}$ & $\begin{array}{c}\text { Амплитудно-фазовая } \\
\text { нестабильность, } \\
\text { дБ }\end{array}$ & $\begin{array}{c}\text { Амплитудная } \\
\text { нестабильность, } \\
\text { дБ }\end{array}$ & $\begin{array}{c}\text { Амплитудно-фазовая } \\
\text { нестабильность, } \\
\text { дБ }\end{array}$ \\
\hline 0,01 & $-73,7$ & $-68,8$ & $-81,2$ & $-71,6$ \\
\hline 0,05 & $-59,4$ & $-55,1$ & $-66,858$ & $-57,5$ \\
\hline 0,1 & $-53,4$ & $-49,0$ & $-60,9$ & $-51,5$ \\
\hline 0,2 & $-47,4$ & $-43,0$ & $-55,1$ & $-45,5$ \\
\hline 0,3 & $-43,9$ & $-39,5$ & $-51,7$ & $-42,0$ \\
\hline
\end{tabular}

\section{Способы улучшения амплитудно-} фазовой стабильности без компенсации

1. При использовании транзисторов без эффектов ловушек (кремниевые биполярные, LDMOS) для обеспечения необходимого уровня амплитудно-фазовой стабильности необходимо подбирать транзисторы по тепловому сопротивлению.

Также для требуемой стабильности необходимо обеспечить питание усилительных каскадов с минимальной девиацией напряжения.

В импульсных СВЧ усилителях мощности в основном применяются блоки питания, рассчитанные на средний ток потребления усилителей. Пиковый ток в импульсе обеспечивается накопительными конденсаторами, ёмкость которых подобрана под требования к максимальной длительности импульса, и током потребления транзисторов в импульсе. Соответственно во время наличия СВЧ импульса конденсаторы разряжаются, а за паузу заряжаются.

Для обеспечения высокой амплитудно-фазовой стабильности необходимо, чтобы за минимальную паузу между импульсами блок питания успевал заряжать конденсаторы до номинального напряжения. В противном случае в последовательных импульсах напряжение питания усилителя будет различаться, что приведёт к ухудшению стабильности. 
2. Отбор транзисторов по тепловому сопротивлению и уровню дефектов у $\mathrm{GaN}$ транзисторов. Для обеспечения требуемого уровня амплитудно-фазовой нестабильности необходимо установить определённую норму на тепловое сопротивление транзисторов и уровень влияния эффекта ловушек.

3. Обеспечение высокого тока транзистора в паузе при низком напряжении стока для транзисторов с эффектом ловушек. Для нивелирования влияния эффекта ловушек на амплитудно-фазовую нестабильность необходимо обеспечить постоянный ток сток-исток транзистора. Но это приведёт к уменьшению КПД на порядок. Для сохранения КПД предлагается поднять напряжение $U_{\text {зи }}$ до предельного значения, вследствие чего увеличится проводимость канала сток-исток. Высокая проводимость канала транзистора позволит сохранить высокий ток в паузе при низком напряжении $U_{\text {си. }}$.

\section{Способы компенсации амплитудно- фазовой стабильности}

Компенсацию изменения амплитуды и фазы от импульса к импульсу можно осуществлять как в составе модуля передатчика, так и в блоке приёма и обработки сигналов РЛС.

В составе модуля можно скорректировать только амплитудно-фазовую нестабильность, определённую детерминированными факторами. Поскольку температуру перехода транзистора, уровень просадки и восстановления напряжения питания во времени можно описать как функцию, зависящую от расстановки выходных импульсов, возможно создать электрическую схему, прогнозирующую изменение фазы и амплитуды выходных радиоимпульсов. Данная схема позволит скорректировать амплитуду при помощи корректировки коэффициента обратной связи узла автоматической регулировки усиления. Фазу можно скорректировать, управляя линейным фазовращателем, тем самым скомпенсировав изменения фазы от импульса к импульсу.

В составе блока приёма и обработки сигналов РЛС можно скорректировать как детерминированные, так и недетерминированные факторы. Если ввести в РЛС второй приёмный тракт, способный записывать сигнал с передатчика, возможна корректировка принимаемого сигнала для исключения амплитудно-фазовой нестабильности передатчика. Данная система является предпочтительной, поскольку устраняет все факторы, влияющие на амплитудно-фазовую нестабильность. Не в каждой РЛС возможно реализовать рассмотренную систему корректировки.

\section{Bblвodbl}

Для обеспечения амплитудно-фазовой нестабильности не более -50 дБ импульсное тепловое сопротивление транзистора должно быть не более 1 К/Вт. Транзистор, рассматриваемый в статье, имеет типовое тепловое сопротивление не более $0,3 \mathrm{~K} / \mathrm{B}$, что соответствует требованиям. Флуктуация напряжения питания транзистора должна быть не более 0,05 В для режима насыщения и не более 0,1 В для линейного режима работы. Для обеспечения соответствия усилительных модулей по параметру амплитудно-фазовой нестабильности в предварительных усилителях установлены линейные стабилизаторы напряжения. Практические измерения показали, что реальная амплитудно-фазовая нестабильность модулей составляет $-51 \ldots-53$ дБ.

В случае несоответствия усилителей требованиям к амплитудно-фазовой нестабильности необходимо применять системы корректировки, описанные в настоящей работе. 


\section{Лuтература}

1. Справочник по радиолокации: в 2-х книгах / Под ред. М.И. Сколника. Книга 1. - М.: Техносфера, $2015.680 \mathrm{c}$.

2. Емельянов, Б.В. Исследование амплитудно-фазовой стабильности биполярных импульсных СВЧ транзисторов, работающих в оконечных усилительных каскадах РЛС / Б.В. Емельянов // Электронная техника. Серия 2. Полупроводниковые приборы. - 2019. Вып. 3 (254). - С. 19-28.

3. Salmer C., Eudeline P., Rolland P.A. Pulse to pulse stability measurement of solid state transmitter module for radar applications // European Microwave Conference, Amsterdam, Netherlands, Oct. 1998. - P. 79-84.

4. Delprato J., Barataud D., Campovecchio M., Neveux G., Tolant C., Eudeline P. Time-domain envelope measurements and simulations of pulseto-pulse stability in microwave power amplifiers // IEEE MTT-S International Microwave Symposium (IMS-2014), pp.1-4.

5. Аронов, В.Л. Моделирование мощного биполярного транзистора в усилительном режиме с учётом квазинасыщения / В.Л. Аронов, А.А. Евстигнеев // Электронная техника. Серия 2. Полупроводниковые приборы. - 2005. - Вып. 1-2. C. 24-33.

\section{References}

1. Spravochnik po radiolokatsii [Handbook on radiolocation]. Moscow, Tekhnosfera, 2015, vol. 1, 680 p.

2. Emelyanov B. V. Issledovaniye amplitudno-fazovoy stabil'nosti bipolyarnykh impul'snykh SVCH tranzistorov, rabotayushchikh $\mathrm{v}$ okonechnykh usilitel'nykh kaskadakh RLS [Research on amplitude-phase of microwave pulsed bipolar transistors of radar output amplifier stages]. Electronic Engineering. Series 2. Semiconductor Devices, 2019, iss. 3(254), pp. 19-28.

3. Salmer C., Eudeline P., Rolland P. A. Pulse to pulse stability measurement of solid state transmitter module for radar applications. European Microwave Conference, Amsterdam, 1998, pp. 79-84.

4. Delprato J., Barataud D., Campovecchio M., Neveux G., Tolant C., Eudeline P. Time-domain envelope measurements and simulations of pulse-to-pulse stability in microwave power amplifiers. IEEE MTT-S International Microwave Symposium (IMS-2014), pp. 1-4.

5. Aronov V. L., Evstigneev A. A. Modelirovaniye moshchnogo bipolyarnogo tranzistora $\mathrm{V}$ usilitel'nom rezhime s uchotom kvazinasyshcheniya [Modeling a power bipolar transistor in an amplifying mode with quasi-saturation]. Electronic Engineering. Series 2. Semiconductor Devices, 2005, iss. 1-2, pp. 24-33. 